1. Giles, R.H., van Es, J.H. \& Clevers, H. Biochim. Biophys. Acta 1653, 1-24 (2003).

2. Veeman, M.T., Axelrod, J.D. \& Moon, R.T. Dev. Cel/5 367-377 (2003).

3. Suzuki, H. et al. Nat. Genet. 36, 417-422 (2004).

4. Suzuki, H. et al. Nat. Genet. 31, 141-149 (2002).

5. Wang, Z., Vogelstein, B. \& Kinzler, K.W. Cancer Res. 63, 5243-5245 (2003).
6. Liu, C. et al. Cell 108, 837-847 (2002).

7. Amit, S. et al. Genes Dev. 16, 1066-1076 (2002).

8. Chan, T.A., Wang, Z., Dang, L.H., Vogelstein, B. \& Kinzler, K.W. Proc. Natl. Acad. Sci. USA 99, 8265-8270 (2002).

9. Sparks, A.B., Morin, P.J., Vogelstein, B. \& Kinzler, K.W. Cancer Res. 58, 1130-1134 (1998).

10. Morin, P.J. et al. Science 275, 1787-1790
(1997)

11. Ilyas, M., Tomlinson, I.P.M., Rowan, A., Pignatelli, M. \& Bodmer, W.F. Proc. Natl. Acad. Sci. USA 94, 10330-10334 (1997)

12. Hart, M.J., de los Santos, R., Albert, I.N., Rubinfeld, B. \& Polakis, P. Curr. Biol. 8, 573-581 (1998).

13. Wehri, M. et al. Nature 407, 527-530 (2000).

\title{
USF1 on trial
}

\author{
Carol C Shoulders
}

\section{The ubiquitous USF proteins regulate the transcription of many genes involved in lipid and glucose homeostasis. A new study provides genetic evidence that USF1 may confer susceptibility to high blood lipid levels.}

Familial combined hyperlipidemia (FCHL), the most common inherited disorder of abnormal blood lipid levels, is believed to share part of its etiology with the metabolic syndrome $^{1}$. The extent of this overlap has been difficult to define because of important differences in diagnosis. FCHL is typically diagnosed through an index individual affected with marked hyperlipidemia (elevated blood cholesterol or triglyceride levels) and normally excludes individuals with hyperlipidemia secondary to other coronary heart disease (CHD) risk factors, such as central obesity and maturity-onset diabetes (type 2 diabetes). More fundamentally, a diagnosis of FCHL demands that index individuals have a blood relative with primary hyperlipidemia, whereas the metabolic syndrome does not. On page $371-376$ of this issue, Päivi Pajukanta and colleagues ${ }^{2}$ present evidence that a specific haplotype of the gene encoding upstream transcription factor 1 (USF1) located in the chromosome 1q21-23 region, is associated with FCHL. They propose that USF1 is a good candidate for conferring susceptibility to the core features of the metabolic syndrome: insulin resistance, glucose intolerance, type 2 diabetes, central obesity, dyslipidemia (elevated blood triglyceride or low high density lipoprotein-cholesterol) and hypertension ${ }^{2}$.

\section{Homing in on USF1 \\ Pajukanta $\mathrm{et} \mathrm{al.}{ }^{3}$ previously found evidence for linkage of the chromosome 1q21-23 interval to FCHL in a cohort of 31 Finnish families, identified through an index individual with early-onset CHD and blood cholesterol or \\ Carol C. Shoulders is in the Genomic of Molecular Medicine Group, MRC Clinical Sciences Centre, Hammersmith Hospital, London, UK. \\ e-mail:carol.shoulders@csc.mrc.ac.uk}

triglyceride levels greater than or equal to ageand sex-specific $90^{\text {th }}$ percentiles. Subsequent studies supported these data and implicated the same chromosomal region in the etiology of type 2 diabetes ${ }^{4-6}$.

In the present study, Pajukanta et al. ${ }^{2}$ built on their previous work by systematically examining a stretch of DNA containing about 10 $\mathrm{Mb}$ for a genetic lesion conferring susceptibility to $\mathrm{FCHL}^{2}$. This involved tracking the transmission of 56 functionally unbiased singlenucleotide polymorphisms (SNPs) to the affected family members of 42 pedigrees with FCHL, including the 31 families who participated in the original linkage study. They examined six SNPs that had some association with FCHL in an additional 18 extended families. The two SNPs that produced the best evidence for association with FCHL are both located in the gene encoding USF1, a transcriptional activator that regulates a number of genes involved in whole-body lipid and glucose homeosta$\operatorname{sis}^{7,8}$. This report prompts two important questions. First, how compelling is the genetic evidence for the involvement of USF1 in FCHL and, by implication, the etiology of the condition? Second, irrespective of the contribution of USF1 to FCHL, do specific USF1 haplotypes increase the risk of type 2 diabetes or the metabolic syndrome?

Regarding the first issue, in the absence of existing linkage data, some investigators ${ }^{9}$ argue for a statistical criterion of $P<5 \times 10^{-9}$, whereas others emphasize the importance of replicating results in independent data sets and of biological plausibility ${ }^{10}$. In the present FCHL study, the alleles at the two associating loci (usf1s1 (exon 11) and usf1s2 (intron 7)) are in strong linkage disequilibrium and, based on family data, have major allele frequencies of about 0.65 in the Finnish population. The lowest $P$ value $\left(9 \times 10^{-7}\right)$ was obtained for the triglyceride trait in men using the gamete- competition test, which views the transmission of alleles or haplotypes to affected individuals as a contest. These data were supported by the results of a second test statistic (HBAT-o) that determined which specific haplotypes were preferentially transmitted. The haplotype containing the minor alleles at the usf1s1 and usfs 2 loci was transmitted less frequently to affected males with the triglyceride trait $\left(P=4 \times 10^{-3}\right)$, prompting the authors to suggest that this might protect against FCHL in their families.

The HBAT-o test also produced evidence $\left(P=7 \times 10^{-4}\right)$ for preferential transmission of the common USF1 haplotype to males with the triglyceride trait of FCHL, supporting the results from three different analyses: the gamete-competition test; a multilocus genoPDT and a haplotype-based haplotype relative risk test. But even robust statistical data from a single cohort of families are not always replicated in another population. Because the Finnish families were, necessarily, selected on the basis of a complex phenotype and did not exclude individuals with type 2 diabetes, the size of the effect of the putative USF1 risk haplotype on any single FCHL-related trait is uncertain. Given the relatively high frequency of the risk haplotype, this effect will presumably vary according to an individual's susceptibility to develop FCHL, which we now know is attributable to at least four other genes ${ }^{11}$.

\section{A SNP in an internal promoter}

The evidence for association of USF1 with FCHL prompted Pajukanta et al. ${ }^{2}$ to sequence this gene in $31 \mathrm{FCHL}$ probands ${ }^{2}$. They found no amino acid changes in USF1 that could account for association of the putative USF1 risk haplotype with FCHL. Nor could they find any difference in USF1 mRNA levels in fat biopsy samples from individuals with FCHL with the risk haplotype versus those without. They did, however, identify a 
putative promoter in intron 7 of USF1. Although the usf1s2 polymorphism, which resides in or close to the putative promoter, had no impact on transcriptional activity, its identification is somewhat encouraging. In principle, the putative promoter could enable USF1 to initiate translation from one of two internal AUGs in exon 8 and, in doing so, throw a switch that reverses the normal function of this protein from activation to repression or vice versa, as has been described for a number of other transcription factors ${ }^{12}$ (Fig. 1). Evidence that the intron 7 promoter is used in vivo will be keenly awaited.

In summary, the work of Pajukanta et al. ${ }^{2}$ has identified USF1 as the prime candidate in the chromosome 1q21-23 linkage region for increasing susceptibility to FCHL, type 2 diabetes and metabolic syndrome. The challenges ahead will be to confirm the genetic data through replication, to identify other genes associated with FCHL at additional loci and to determine the roles of the different isoforms of USF1 in the regulation of genes pivotal for whole-body lipid and glucose homeostasis and the maintenance of the arterial wall vasculature ${ }^{13}$.

1. Lewis, G.F. Curr. Opin. Lipidol. 13, 97-99 (2002).

2. Pajukanta, P. et al. Nat. Genet. 36, 371-376 (2004)

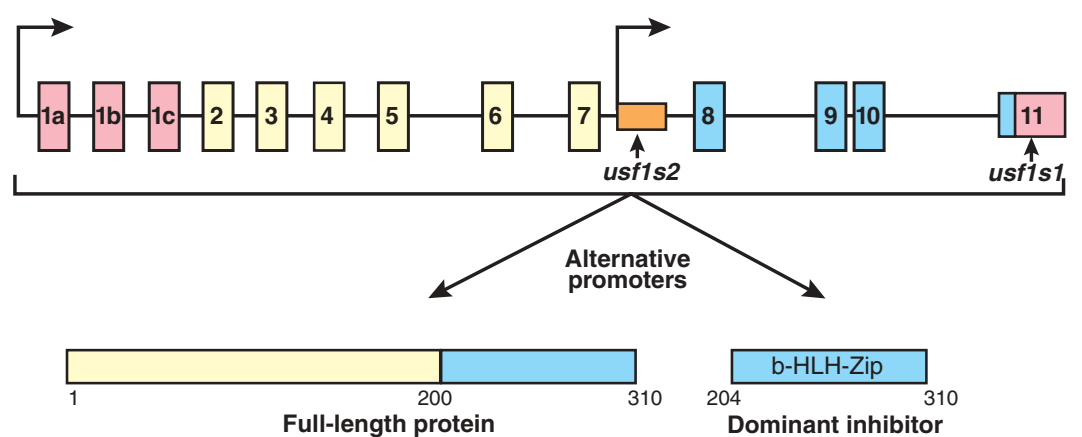

Figure 1 A theoretical scenario to illustrate how the use of a putative promoter in intron 7 of USF1 (orange box) might lead to the generation of a mini-USF1 protein lacking the transactivation domain. Because mini-USF proteins in vitro behave as transdominant inhibitors ${ }^{14,15}$, there is a substantial incentive to establish whether the putative promoter identified by Pajukanta et l. $^{2}$ might operate in vivo to downregulate USF1 activity. The two polymorphisms associated with FCHL in Finnish families are indicated. b-HLH-Zip, basic helix-loop-helix-leucine zipper.

3. Pajukanta, P. et al. Nat. Genet. 18, 369-373 (1998).

4. Pei, L. J. Biol. Chem. 276, 8484-8491 (2001).

5. Coon, H. et al. Arterioscler. Thromb. Vasc. Biol. 20, 2275-2280 (2000).

6. McCarthy, M.I. Curr. Diab. Rep. 3, 159-167 (2003).

7. Smih, F. et al. Diabetes 51, 293-300 (2002).

8. Moore, M.L., Park, E.A. \& McMillin, J.B. J. Biol. Chem. 278, 17263-17268 (2003).

9. Risch, N. \& Merikangas, K. Science 273, 1516-1517 (1996)

10. Page, G.P., George, V., Go, R.C., Page, P.Z. \& Allison, D.B. Am. J. Hum. Genet. 73, 711-719 (2003).
11. Shoulders, C.C., Jones, E.L. \& Naoumova, R.P. Hum. Mol. Genet. 13, R149-R160 (2004).

12. Foulkes, N.S. \& Sassone-Corsi, P. Cel/ 68, 411-414 (1992).

13. Chen, Y.H., Layne, M.D., Watanabe, M., Yet, S.F. \& Perrella, M.A. J. Biol. Chem. 276, 47658-47663 (2001).

14. Lefrancois-Martinez, A.M., Martinez, A., Antoine, B., Raymondjean, M. \& Kahn, A. J. Biol. Chem. 270, 2640-2643 (1995).

15. Viollet, B. et al. J. Biol. Chem. 271, 1405-1415 (1996)

\title{
Phenotype matters
}

\author{
Leslie G Biesecker
}

\section{The association of diseases with genes is complex, even among mendelian disorders. A new study shows that mutations in the gene encoding filamin B (FLNB) cause four distinct disorders of human skeletal development.}

\begin{abstract}
Mapping a mendelian disorder to a genetic locus is easier than determining the relationships between genes and disorders or between different disorders. Initially, it seemed that the correspondence of diseases to genes would be simple: one gene, one disease. As we have learned from positional cloning efforts, this is often untrue. But, as a new study by Deborah Krakow and colleagues ${ }^{1}$ illustrates, the reality is far more interesting.

They found that mutations in FLNB, encoding filamin $\mathrm{B}$, which belongs to a family of proteins that act as key regulators of the actin cytoskeleton, underlie four distinct

Leslie G. Biesecker is at the National Human Genome Research Institute, National Institutes of Health, Bethesda, Maryland. e-mail: leslieb@helix.nih.gov
\end{abstract}

human skeletal disorders ${ }^{1}$. The implications of this work are many, and two in particular deserve attention.

\section{Different vantage points}

From a clinical genetics standpoint, spondylocarpotarsal syndrome (OMIM 272460), Larsen syndrome (OMIM 150250) and atelosteogenesis types I (AOI; OMIM 108720) and III (AOIII; OMIM 108721) are four distinct human disorders. In contrast, a basic scientist may view them as a single disorder of filamin B dysfunction with inconsequential phenotypic differences. Both perspectives are valid, but they may result in incompatible classification schemes that impede a comprehensive understanding of human genetic disease.

The determination of allelism does not supplant phenotypic distinctions. Although AOI and AOIII share some features, the phenotypic distinctions are important. The qualitative differences in phenotypic manifestations that distinguish atelosteogenesis from spondylocarpotarsal and Larsen syndromes tell us something about filamin B biology. This information follows on the heels of a report describing mutations in a related gene, FLNA, as the cause of five distinct disorders in humans $^{2}$ (otopalatodigital syndromes types I and II, OMIM 311300 and 304120, respectively; Melnick-Needles syndrome, OMIM 309350; periventricular nodular heterotopia, OMIM 300049; and frontometaphyseal dysplasia, OMIM 305620). This is interesting because there are overlapping phenotypic features in the disorders associated with FLNA and FLNB (Fig. 1). Furthermore, Larsen syndrome probably has genetic heterogeneity, as 